\title{
Variation of the $90^{\circ}$ Redundant-hole Diameter Effect on the Response and Failure of Round-hole 6061-T6 Aluminum Alloy Tubes under the Cyclic Bending
}

\author{
Kuo-Long Lee ${ }^{1}$, Wen-Fung Pan $^{2 *}$ \\ 1. Department of Innovative Design and Entrepreneurship Management, Far East University, Tainan 744, \\ Taiwan \\ 2. Department of Engineering Science, National Cheng Kung University, Tainan 701, Taiwan \\ E-mail: z7808034@email.ncku.edu.tw (Corresponding author)
}

Received: 15 July 2020; Accepted: 7 September 2020; Available online: 20 September 2020

\begin{abstract}
This paper presents an experiment and an analysis for examining the variation of the $90^{\circ}$ redundanthole diameter effect on the cyclic bending mechanical behavior and fracture failure of round-hole 6061-T6 aluminum alloy tubes. In this investigation, a round-hole 6061-T6 aluminum alloy tube with a $6 \mathrm{~mm}$ hole diameter was drilled to obtain a $90^{\circ}$ redundant hole but with different hole diameter of $2,4,6$, 8 , or $10 \mathrm{~mm}$. It can be observed that from the first bending cycle, the bending moment-curvature curve describes a stable loop. The diameter of the $90^{\circ}$ redundant hole has little effect on the bending moment-curvature relationship. However, when the number of bending cycles increases, the ovalization-curvature curve shows an increasing, asymmetrical, ratcheting, and bow-like tendency. The diameter of $90^{\circ}$ redundant-hole shows a significant influence on the ovalization-curvature relationship. In addition, five non-parallel straight lines corresponding to five different $90^{\circ}$ redundant hole diameters were discovered for the controlled curvature-number of bending cycles necessary to cause failure relationship on the double logarithmic coordinates. Finally, a formula was presented to simulate the above relationship. It is found that the experimental and analytical data were in good agreement.

Keywords: Round-hole 6061-T6 aluminum alloy tubes; $90^{\circ}$ redundant hole; Redundant-hole diameter; Cyclic bending; Moment; Curvature; Ovalization; Failure.
\end{abstract}

\section{Introduction}

Round-hole tube (abbreviation: RHT) is a tube drilled with a round hole. RHT is usually used as a connection for automobile, motorcycle or bicycle parts. When RHT bears a bending load, the circular cross section will gradually become elliptical as the degree of bending increases. The bending stiffness of the RHT will gradually decrease, which is called deterioration. A quantity "ovalization" is used to describe the deterioration, which is the decrease in the outer diameter $\left(\Delta D_{0}\right)$ divided by the original outer diameter $\left(D_{0}\right) . \Delta D_{0} / D_{0}$ increases with the number of cycles in cyclic bending load. Eventually, RHT will suffer fracture damage when a certain number of cycles is attained.

So far, the research on the cyclic bending of smooth circular tubes has achieved important results. In 1987, Professor Kyriakides and his research team designed a mechanical device for cyclic bending tests on various round pipes (6061-T6 aluminum alloy pipe, 1018 steel pipe, 304 stainless steel pipe, 1020 steel pipe and NiTi pipe) with or without internal or external pressure, many experiments and theoretical studies have been conducted under monotonic or cyclic bending [1-6]. In addition, many scholars have also presented related research. Elchalakani et al. [7] carried out experimental tests on C350 steel pipes with different diameter/thickness ratios $\left(D_{\mathrm{o}} / t\right.$ ratio) under bending. Jiao and Zhao [8] examined the bending response of very-high-strength steel pipes. Houliara and Karamanos [9] studied the in-phase bending buckling of pressured thin-walled tubes. Yazdani and Nayebi [10] studied the fatigue damage and ratcheting behavior of thin-walled pipes subjected to cyclic bending with a stable internal pressure. By using the measured strain in the plastic bending test, the new ductile slenderness limits of the CFT structural plastic design was determined by Elchalakani et al. [11]. Li and Wang [12] studied the stability of reinforced single-layer lattice shells under earthquake action. Chegeni et al. [13] explored the influence of corrosion shape and depth on the response of internal pressure bending pipes.

In 2010, Pan et al. began to study the response of notched tubes under cyclic bending loads. Lee et al. [14] studied the cyclic bending change of $\Delta D_{0} / D_{0}$ of sharp-notched circular tubes. Lee et al. [15] discussed the rupture of sharp-notched SUS304 stainless steel pipes periodically bent under different curvatures. This work also studied 
the changes in curvature and notch depth. Chung et al. [16] studied the cyclic bending stability of the sharp-notched 6061-T6 aluminum alloy tube. However, all of the above-mentioned studies are for circumferential notched tubes. Thereafter, Lee et al. [17] inspected the cyclic bending degradation and failure of 6061-T6 aluminum alloy tubes with local sharp cuts. In addition, the relationship between bending moment $(M)$-curvature $(\kappa)$ and $\Delta D_{0} / D_{0}-\kappa$ was analyzed by ANSYS. Lee et al. [18] studied the cyclic bending response of SUS304 stainless steel pipes with local sharp grooves. In their research, ANSYS also analyzed the $M-\kappa$ and $\Delta D_{0} / D_{0}-\kappa$ relationships.

In this study, the effect of $90^{\circ}$ redundant hole diameter on the response and failure of round-hole 6061-T6 aluminum alloy tubes (abbreviation: Al 6061-T6 RHTs) subjected to cyclic bending was investigated. Al 6061-T6 RHT with a $6 \mathrm{~mm}$ hole diameter was drilled to obtain a $90^{\circ}$ redundant hole but with different hole diameter of 2 , 4,6 , 8 , or $10 \mathrm{~mm}$. The experimental study is a cyclic bending test of curvature control. The $M, \kappa$ and $\Delta D_{0} / D_{0}$ were measured by the test device. In addition, the number of bending cycles necessary to cause failure $\left(N_{\mathrm{f}}\right)$ was also recorded.

\section{Experiments}

\subsection{Experimental devices}

Fig. 1 schematically depicts an experiment performed by a dedicated tube bender. The equipment is set up for monotonic and cyclic bending tests. The work of Pan et al. [19] includes a detailed description of the experimental setup. In addition, Pan et al. [19] designed a new device to measure $\kappa$ and $\Delta D_{0} / D_{0}$ of the tube shown in Fig. 2 . The two side-inclinometers in the equipment are used to detect the angle change of the pipe during the cyclic bending process. Based on the angle change, the $\kappa$ can be obtained by simple calculation. The calculated version can be discovered in the paper by Pan et al. [19]. The $\Delta D_{0} / D_{\text {o }}$ can also be measured in the central part of the device including the magnetic detector and the magnetic block.

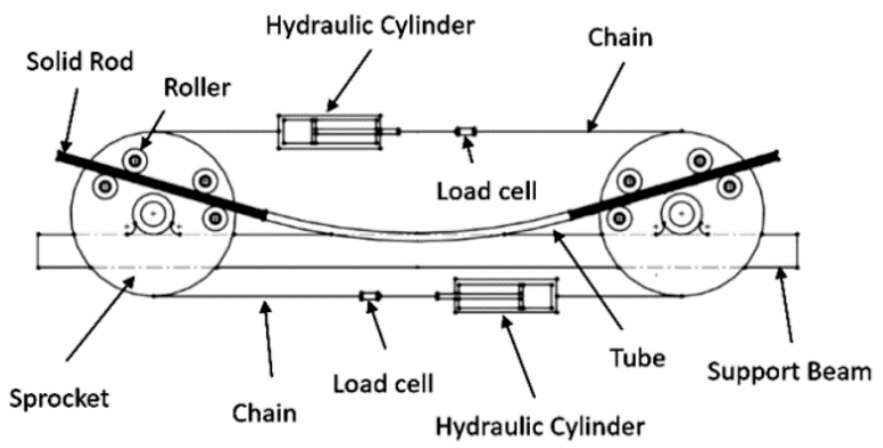

Fig. 1. The tube bender

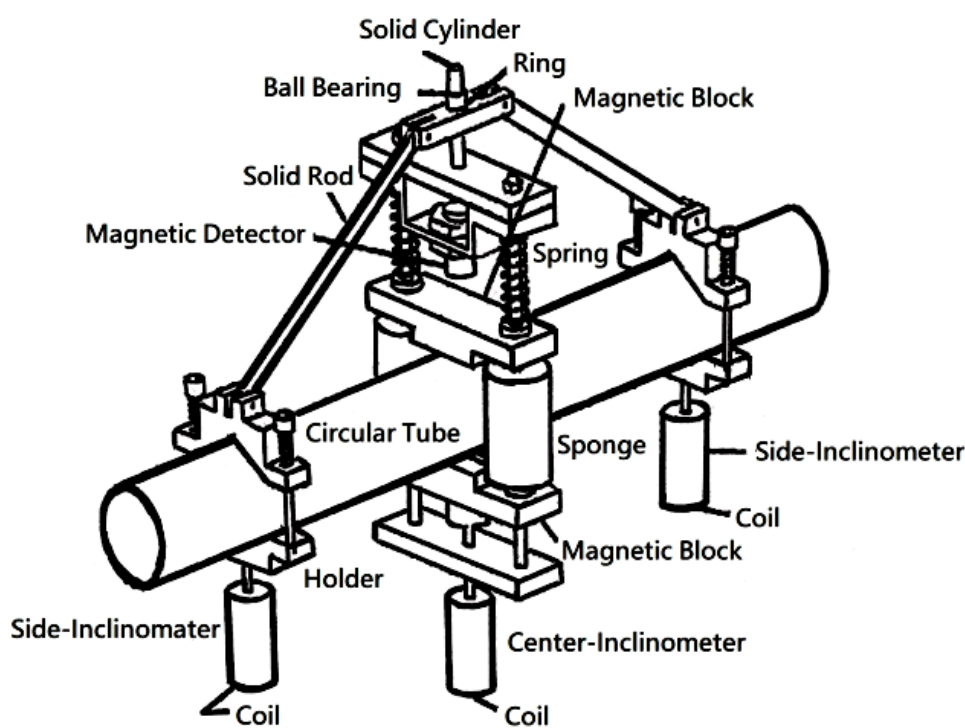

Fig. 2. The curvature-ovalization measurement device 


\subsection{Material and specimens}

The 6061-T6 aluminum alloy round tube is used in this study. Its chemical composition (weight percentage) is Mg 0.916, Si 0.733, Cu 0.293, Ti 0.268, Fe 0.256, Mn 0.132, Zn 0.0983, Cr 0.0682, Ni 0.0056, and Pb 0.005, Sn $<0.001$ and $\mathrm{Al}$ remains. The mechanical properties of the material are: yield stress of $282 \mathrm{MPa}$, ultimate tensile stress of $313 \mathrm{MPa}$, and percentage elongation of $13 \%$.

In this study, we drilled the original 6061-T6 aluminum alloy tube with outer diameter $\left(D_{0}\right)$ of $35.0 \mathrm{~mm}$ and wall thickness $(t)$ of $3.0 \mathrm{~mm}$ to obtain RHT with a $6 \mathrm{~mm}$-hole-diameter. Next, we drilled a $\phi=90^{\circ}$ redundant hole on the same cross section, and its redundant hole diameter $(d)$ was 2, 4, 6, 8 or $10 \mathrm{~mm}$. Fig. 3 demonstrates a schematic diagram of the RHT with a $\phi$ redundant hole, and Fig. 4 depicts the dimensions of Al 6061-T6 RHT with a $\phi=90^{\circ}$ redundant hole. Note that the same cross section is the A-A cross section. As shown in Fig. 3, the direction of the round hole on the RHT is the $y$ direction. Since the cyclic bending moment is in the $z$ direction, the cyclic bending causes the most serious deterioration and damage to the RHT.

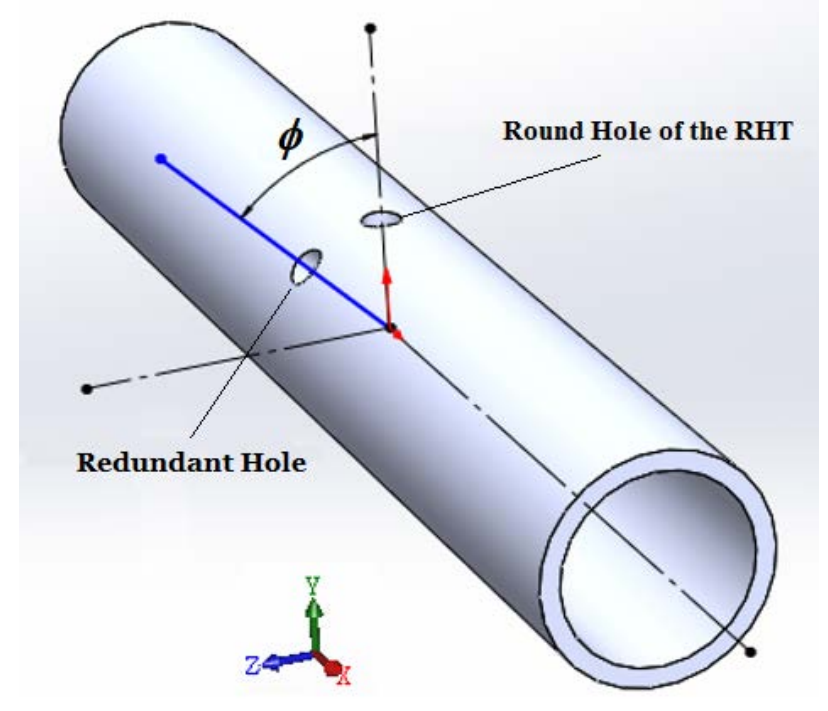

Fig. 3. A schematic drawing of a RHT with a $\phi$ redundant hole

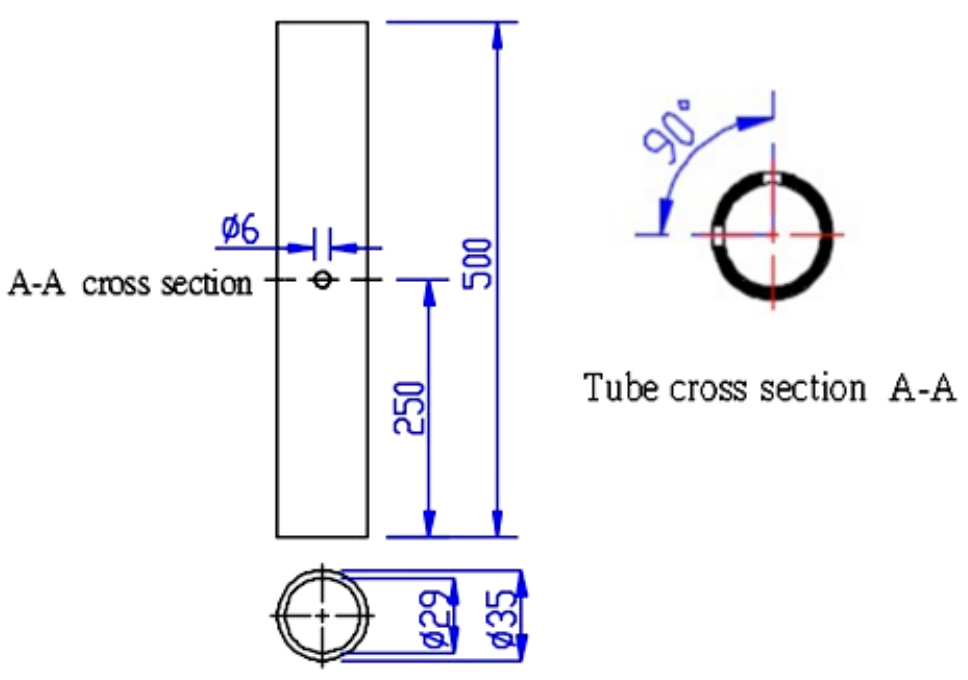

Fig. 4. Dimensions of the $\mathrm{Al} 6061-\mathrm{T} 6 \mathrm{RHT}$ with a $\phi=90^{\circ}$ redundant hole

\subsection{Test procedures}

The experiment was a curvature controlled cyclic bending test, and the curvature rate was a constant at 0.03 $\mathrm{m}^{-1} \mathrm{~s}^{-1}$. The $M$ is measured by two load cells (Fig. 1) installed in the pipe bender. As shown in Fig. 2, the $\kappa$ and $\Delta D_{0} / D_{0}$ were measured by a curvature-ovalization measurement device. The $N_{\mathrm{f}}$ was also recorded. 


\section{Results and discussion}

\subsection{Mechanical behavior}

Fig. 5 depicts an experimental cyclic $M-\kappa$ curve for Al 6061-T6 RHT with a $\phi=90^{\circ}$ and $d=6 \mathrm{~mm}$ redundant hole under cyclic bending. It can be observed that from the first bending cycle, the $M-\kappa$ curve describes a stable loop. Since the redundant holes were small and local, the $M-\kappa$ curves for Al 6061-T6 RHTs with $\phi=90^{\circ}$ and different $d$ redundant holes under cyclic bending were almost the same. Therefore, this paper does not show the $M-\kappa$ curves for Al 6061-T6 RHTs with $\phi=90^{\circ}$ and different $d$ redundant holes under cyclic bending.

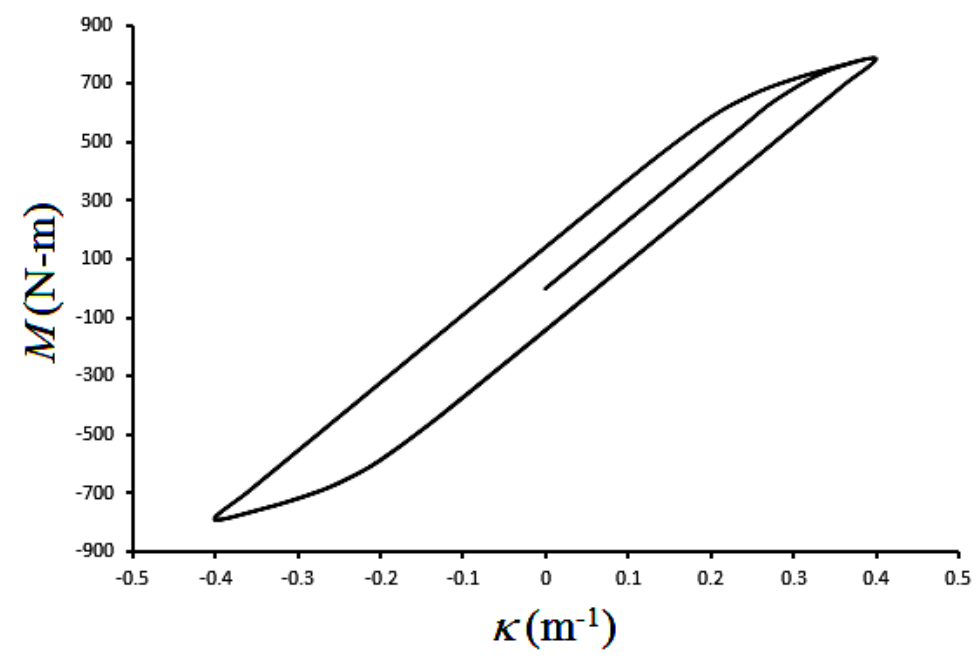

Fig. 5. Experimental $M-\kappa$ curve for Al 6061-T6 RHT with a $\phi=90^{\circ}$ and $d=6 \mathrm{~mm}$ redundant hole under cyclic bending.

Figs. 6(a)-6(c) respectively depict the experimental cyclic $\Delta D_{0} / D_{0}-\kappa$ relationships for Al 6061-T6 RHTs with $\phi=90^{\circ}$ and $d=2,6$ and $10 \mathrm{~mm}$ redundant holes subjected to cyclic bending. It is observed that as the number of bending cycles increases, the $\Delta D_{0} / D_{0}-\kappa$ relationship shows an increasing, asymmetrical, ratcheting, and bow-like tendency. In addition, when the round hole is under tension, $\Delta D_{0} / D_{0}$ continues to increase. However, when the round hole is under compression, $\Delta D_{0} / D_{0}$ continues to decrease.

\subsection{Fracture failure}

Fig. 7 presents the experimental controlled curvature $\left(\kappa_{\mathrm{c}} / \kappa_{0}\right)-N_{\mathrm{f}}$ relationships for Al 6061-T6 RHTs with $\phi=$ $90^{\circ}$ and different $d$ redundant holes under cyclic bending. Note that the $\kappa_{\mathrm{c}}$ was normalized by $\kappa_{0}=t / D_{0}{ }^{2}$. Next, the data in Fig. 7 was plotted on double logarithmic coordinates, and five non-parallel straight lines were found in Fig. 8. Note that these lines were obtained by the method of least squares.

In our research, the failure type was fatigue fracture. Therefore, the empirical formula proposed by Kyriakides and Shaw [1] was modified to:

$$
\kappa_{\mathrm{C}} / \kappa_{\mathrm{o}}=C\left(N_{\mathrm{f}}\right)^{-\alpha} \quad \text { or } \quad \log \kappa_{\mathrm{C}} / \kappa_{\mathrm{o}}=\log C-\alpha \log N_{\mathrm{f}} .
$$

Here, $C$ is the value of $\kappa_{\mathrm{C}} / \kappa_{\mathrm{o}}$ when $N_{\mathrm{f}}$ is equal to 1 , and $\alpha$ is the slope of the straight line of the log-log graph. According to the experimental data in Fig. 8, five magnitudes of $C$ were respectively obtained for $d=2,4,6,8$ and $10 \mathrm{~mm}$ from Eq. (1b) to be 0.331, 0.312, 0.356, 0.310 and 0.320. According to the experimental data in Fig. 8, five magnitudes of $\alpha$ were respectively determined for $d=2,4,6,8$ and $10 \mathrm{~mm}$ from Eq. (1b) to be $0.165,0.152$, 0.174, 0.159 and 0.163. Fig. 9 depicts the relationship between $C$ and $d / t$. The following formulation was proposed:

$$
C=c_{1}-c_{2} \sin \left(c_{3}(d / t-0.667) 180^{\circ}\right)
$$

where $c_{1}, c_{2}$ and $c_{3}$ are material parameters. When the $d / t$ value is 0.667 , the value of $c_{1}$ can be determined to be 0.331. The value of $c_{2}$ is the amplitude of the periodic function and the value of $c_{3}$ is the parameter of the correction period. Therefore, from Fig. 9, the values of $c_{2}$ and $c_{3}$ can be respectively determined to be 0.025 and 1.125. Fig. 10 depicts the relationship between $\alpha$ and $d / t$. The following formulation was proposed: 


$$
\alpha=a_{1}-a_{2} \sin \left(a_{3}(d / t-0.667) 180^{\circ}\right)
$$

where $a_{1}, a_{2}$ and $a_{3}$ are material parameters. When the $d / t$ value is 0.667 , the value of $a_{1}$ can be determined to be 0.165 . The value of $a_{2}$ is the amplitude of the periodic function and the value of $a_{3}$ is the parameter of the correction period. Therefore, from Fig. 10, the values of $a_{2}$ and $a_{3}$ can be respectively obtained to be 0.012 and 1.132. Finally, using Eqs. (1), (2), and (3), the simulated $\kappa_{\mathrm{c}} / \kappa_{0}-N_{\mathrm{f}}$ relationships for Al 6061-T6 RHTs with $\phi=90^{\circ}$ and different $d$ redundant holes under cyclic bending on double logarithmic coordinates are demonstrated in solid lines in Figs. 11-15. A good agreement has been reached between experimental results and simulation results.

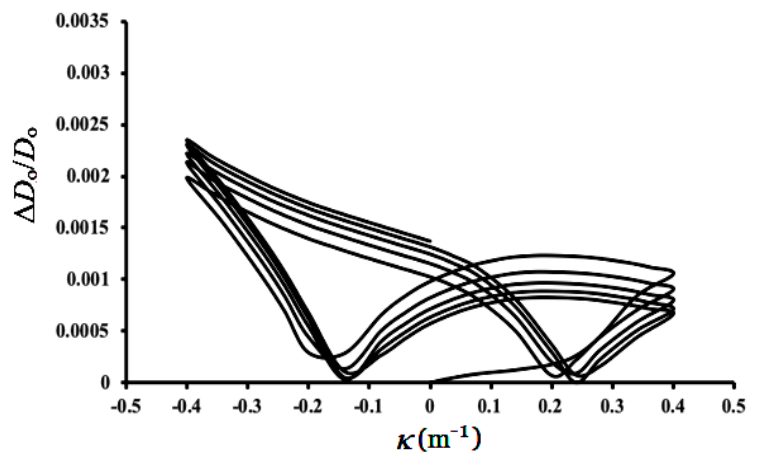

(a)

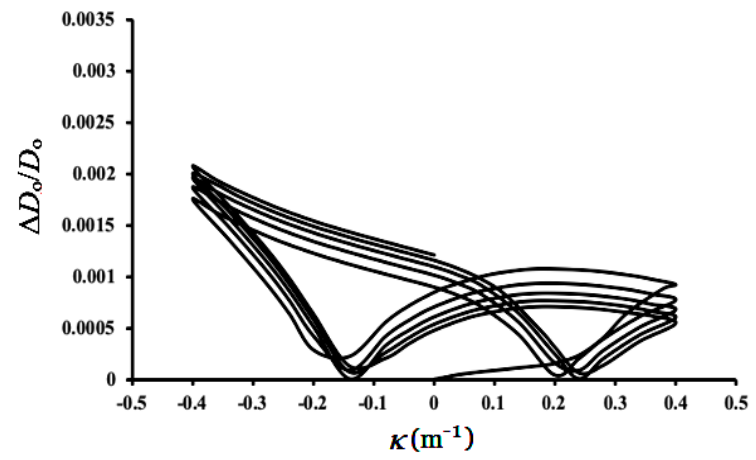

(b)

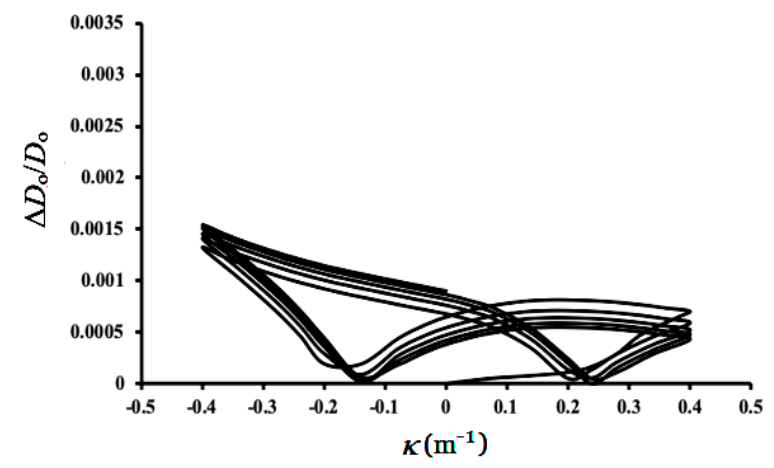

(c)

Fig. 6. Experimental $\Delta D_{0} / D_{0}-\kappa$ curves for Al 6061-T6 RHTs with $\phi=90^{\circ}$ and $d=$ (a) 2, (b) 6 and (c) $10 \mathrm{~mm}$ redundant holes under cyclic bending

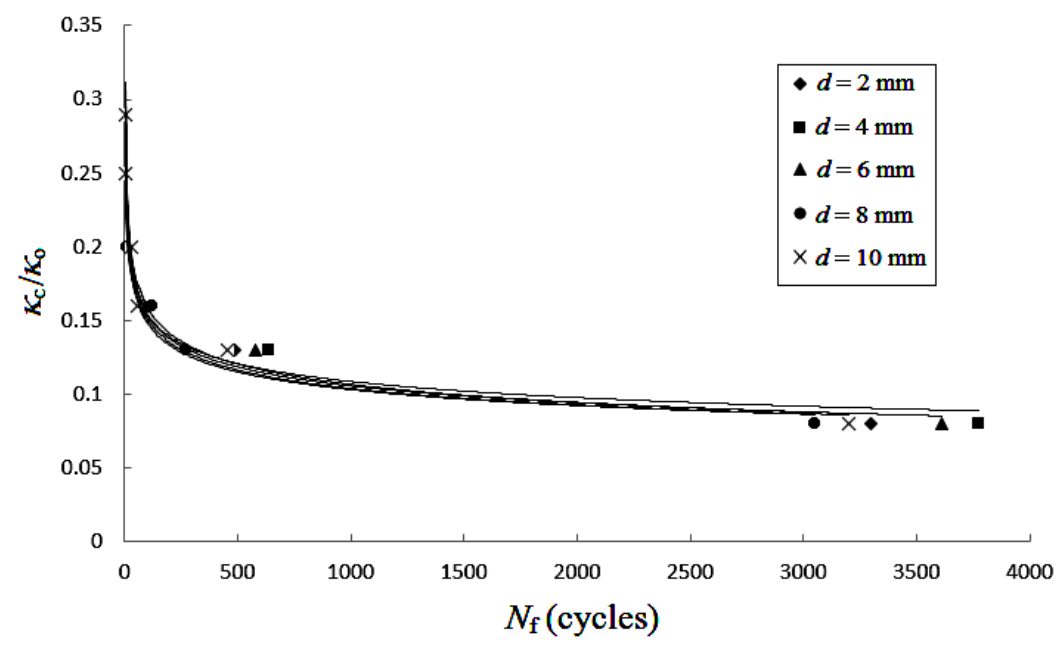

Fig. 7. Experimental $\kappa_{\mathrm{c}} / \kappa_{\mathrm{o}}-N_{\mathrm{f}}$ curves for Al 6061-T6 RHTs with $\phi=90^{\circ}$ and different $d$ redundant holes under cyclic bending on decimal coordinates 


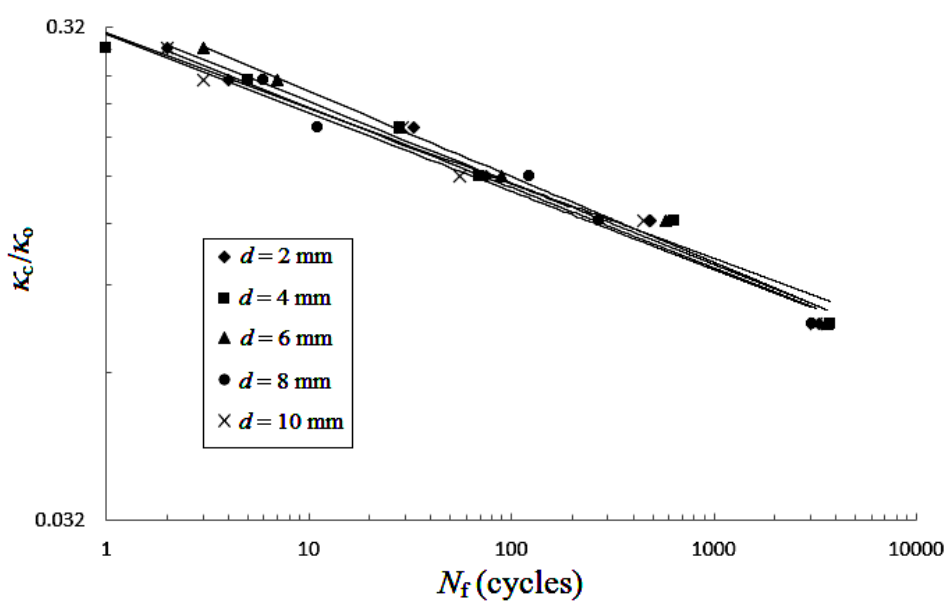

Fig. 8. Experimental $\kappa_{\mathrm{c}} / \kappa_{\mathrm{o}}-N_{\mathrm{f}}$ curves for Al 6061-T6 RHTs with $\phi=90^{\circ}$ and different $d$ redundant holes under cyclic bending on double logarithmic coordinates

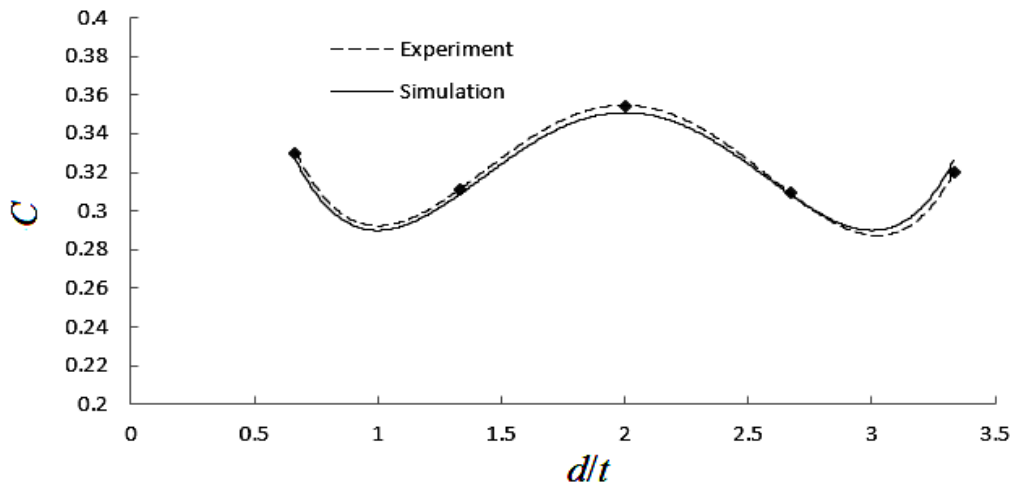

Fig. 9. Experimental and simulated relationships between $C$ and $d / t$

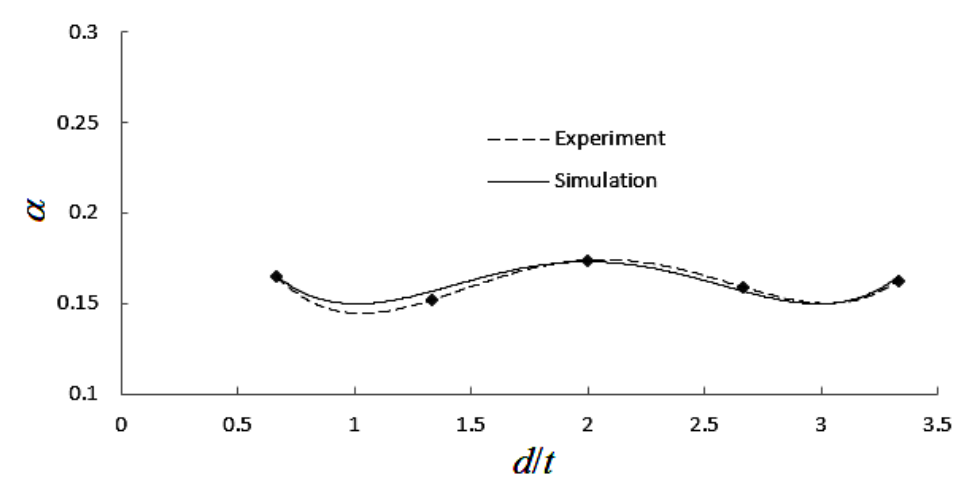

Fig. 10. Experimental and simulated relationships between $\alpha$ and $d / t$

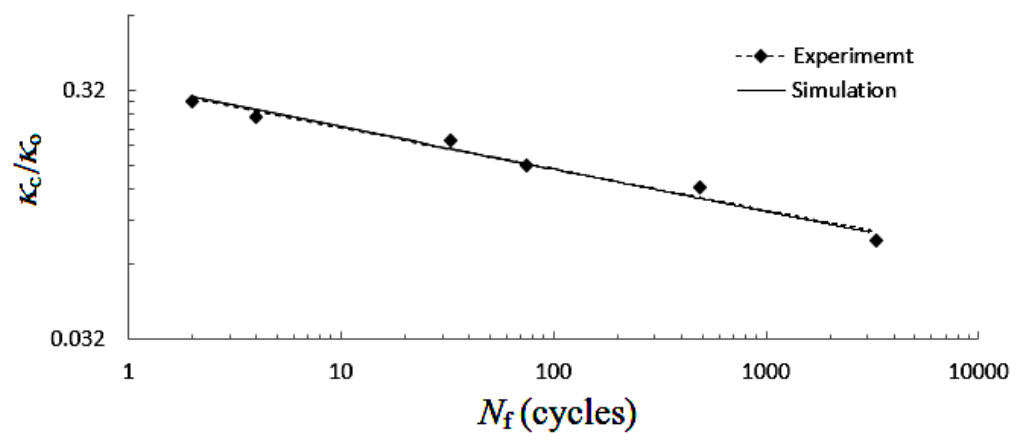

Fig. 11. Experimental and simulated $\kappa_{\mathrm{C}} / \kappa_{0}-N_{\mathrm{f}}$ curves for Al 6061-T6 RHTs with a $\phi=90^{\circ}$ and $d=2$ mm redundant hole under cyclic bending on double logarithmic coordinates 


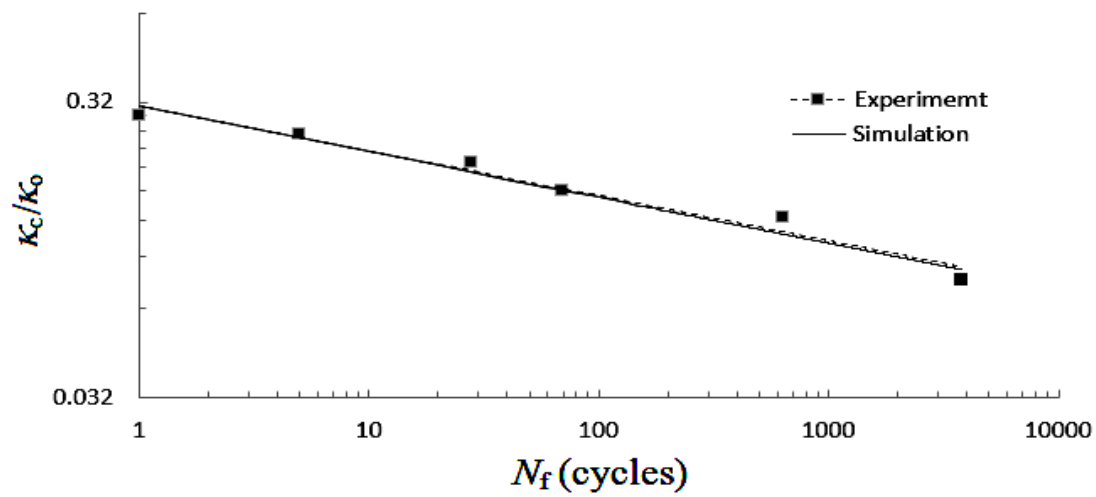

Fig. 12. Experimental and simulated $\kappa_{\mathrm{C}} / \kappa_{0}-N_{\mathrm{f}}$ curves for $\mathrm{Al}$ 6061-T6 RHTs with a $\phi=90^{\circ}$ and $d=4$ mm redundant hole under cyclic bending on double logarithmic coordinates

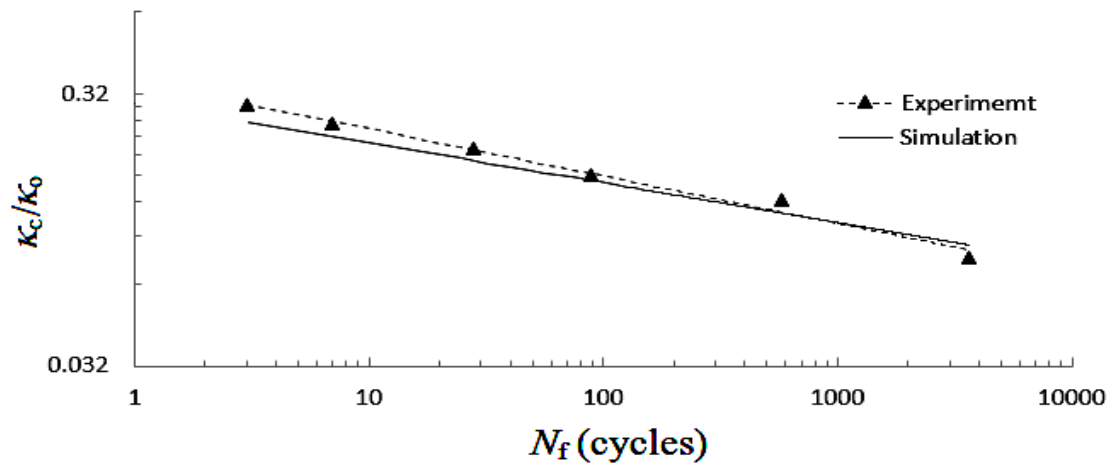

Fig. 13. Experimental and simulated $\kappa_{\mathrm{C}} / \kappa_{0}-N_{\mathrm{f}}$ curves for Al 6061-T6 RHTs with a $\phi=90^{\circ}$ and $d=6$ mm redundant hole under cyclic bending on double logarithmic coordinates

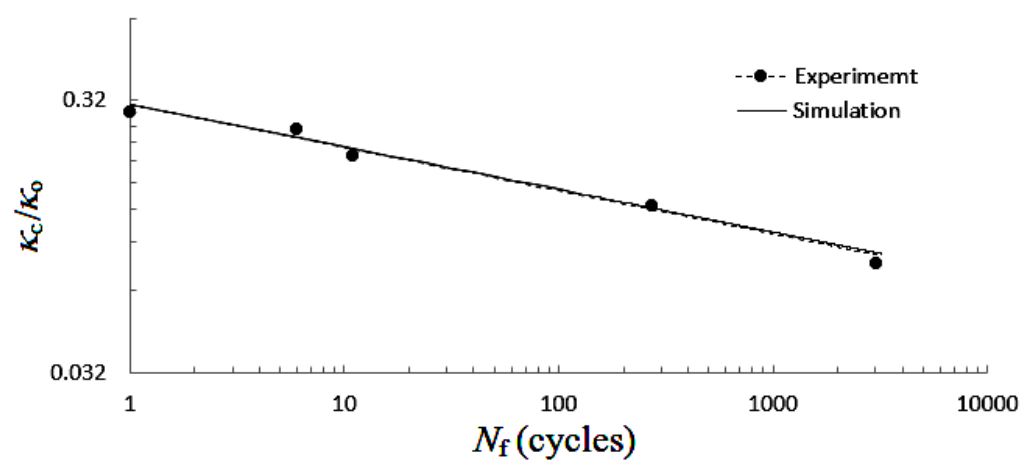

Fig. 14. Experimental and simulated $\kappa_{\mathrm{c}} / \kappa_{0}-N_{\mathrm{f}}$ curves for $\mathrm{Al}$ 6061-T6 RHTs with a $\phi=90^{\circ}$ and $d=8 \mathrm{~mm}$ redundant hole under cyclic bending on double logarithmic coordinates

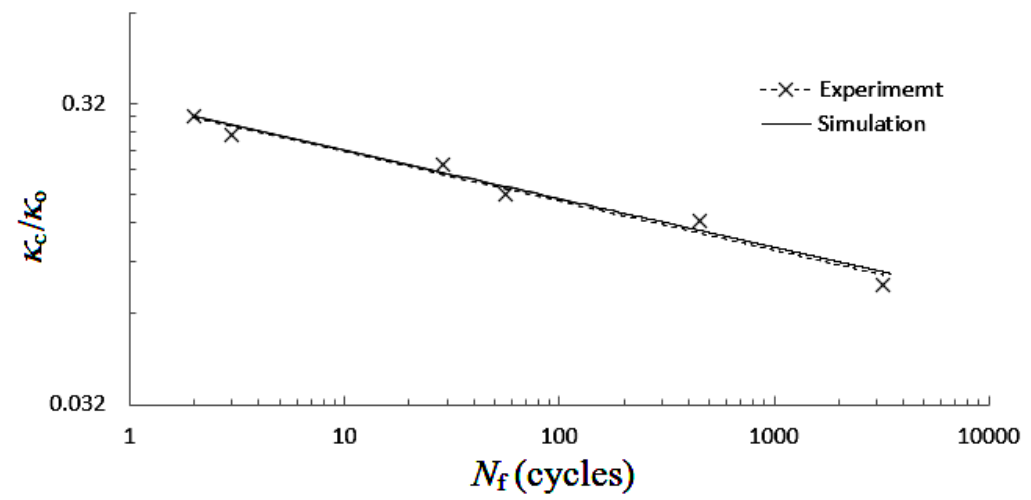

Fig. 15. Experimental and simulated $\kappa_{\mathrm{C}} / \kappa_{\mathrm{o}}-N_{\mathrm{f}}$ curves for Al 6061-T6 RHTs with a $\phi=90^{\circ}$ and $d=10$ mm redundant hole under cyclic bending on double logarithmic coordinates 


\section{Conclusions}

The response and failure of Al 6061-T6 RHTs with $\phi=90^{\circ}$ and different $d$ redundant holes subjected to cyclic bending were explored. Based on the results of experiments and simulations, this study draws the following conclusions:

(1) The experimental $M-\kappa$ relationships for Al 6061-T6 RHTs with $\phi=90^{\circ}$ and different $d$ redundant holes subjected to cyclic bending display stable loops from the first bending cycle. Since the redundant holes were small and local, the $M-\kappa$ curves for Al 6061-T6 RHTs with $\phi=90^{\circ}$ and different $d$ redundant holes under cyclic bending were almost the same.

(2) The experimental $\Delta D_{0} / D_{0}-\kappa$ relationships for Al 6061-T6 RHTs with $\phi=90^{\circ}$ and different $d$ redundant holes subjected to cyclic bending reveal increasing, asymmetrical, ratcheting, and bow-like tendency with the increase in the number of bending cycles. In addition, $\Delta D_{\mathrm{o}} / D_{\mathrm{o}}$ continues to increase when the round hole is under tension. However, $\Delta D_{\mathrm{o}} / D_{\mathrm{o}}$ continues to decrease when the round hole is under compression.

(3) The empirical formula proposed by Kyriakides and Shaw [1] was modified to describe the $\kappa_{\mathrm{c}} / \kappa_{\mathrm{0}}-N_{\mathrm{f}}$ relationships for $\mathrm{Al}$ 6061-T6 RHTs with $\phi=90^{\circ}$ and different $d$ redundant holes submitted to cyclic bending. Based on the experimental data, the material parameters $C$ and $\alpha$ were proposed in Eqs. (2) and (3), respectively. Additionally, the analysis data obtained by Eqs. (1)-(3) were very close to the experimental results (Figs. 11-15).

\section{Acknowledgement}

This research was carried out with the support of the Ministry of Science and Technology, and the research project was MOST 106-2221-E-006-097. We sincerely thank them for their assistance.

\section{References}

[1] Kyriakides S, Shaw PK. Inelastic buckling of tubes under cyclic loads. Journal of Pressure Vesels and Technology. 1987;109(2):169-178.

[2] Corona E, Kyriakides S. An experimental investigation of the degradation and buckling of circular tubes under cyclic bending and external pressure. Thin-Walled Structures. 1991;12(3):229-263.

[3] Corona E, Lee LH, Kyriakides S. Yield anisotropic effects on buckling of circular tubes under bending. International Journal of Solids and Structures. 2006;43(22):7099-7118.

[4] Limam A, Lee LH, Kyriakides S. On the collapse of dented tubes under combined bending and internal pressure. International Journal of Solids and Structures. 2012;55(1):1-12.

[5] Bechle NJ, Kyriakides S. Localization of NiTi tubes under bending. International Journal of Solids and Structures. 2014;51(5):967-980.

[6] Jiang D, Kyriakides S, Bechle NJ, Landis CM. Bending of pseudoelastic NiTi tubes. International Journal of Solids and Structures. 2017;124:192-214.

[7] Elchalakani M, Zhao XL, Grzebieta RH. Plastic mechanism analysis of circular tubes under pure bending. International Journal of Mechanical Sciences. 2002;44(6):1117-1143.

[8] Jiao H, Zhao XL. Section slenderness limits of very high strength circular steel tubes in bending. Thin-Walled Structures. 2004;42(9):1257-1271.

[9] Houliara S, Karamanos SA. Buckling and post-buckling of long pressurized elastic thin-walled tubes under in-plane bending. International Journal of Nonlinear Mechanics. 2006;41(4):491-511.

[10] Yazdani H, Nayebi A. Continuum damage mechanics analysis of thin-walled tubes under cyclic bending and internal constant pressure. International Journal of Applied Mechanics. 2013;5(4):1350038.

[11] Elchalakani M, Karrech A, Hassanein MF, Yang B. Plastic and yield slenderness limits for circular concrete filled tubes subjected to static pure bending. Thin-Walled Structures. 2016;109:50-64.

[12] Li P, Wang L. Nonlinear stability behavior of cable-stiffened single-layer latticed shells under earthquakes. International Journal of Structural Stabability and Dynamics. 2018;18(10):1850117.

[13] Chegeni B, Jayasuriya S, Das S. Effect of corrosion on thin-walled pipes under combined internal pressure and bending. Thin-Walled Structures. 2019;143:106218.

[14] Lee KL, Hung CY, Pan WF. Variation of ovalization for sharp-notched circular tubes under cyclic bending. Journal of Mechanics. 2010;26(3):403-411.

[15] Lee KL, Hsu CM, Pan WF. Viscoplastic collapse of sharp-notched circular tubes under cyclic bending. Acta Mechanica Solida Sinica. 2013;26(6):629-641.

[16] Chung CC, Lee KL, Pan WF. Collapse of sharp-notched 6061-T6 aluminum alloy tubes under cyclic bending. International Journal of Structural Stability and Dynamics. 2016;26(7):1550035. 
[17] Lee KL, Chang KH, Pan WF. Failure life estimation of sharp-notched circular tubes with different notch depths under cyclic bending. Structural Engineering and Mechanics. 2016;60(3):387-404.

[18] Lee KL, Chang KH, Pan WF. Effects of notch depth and direction on stability of local sharp-notched circular tubes subjected to cyclic bending. International Journal of Structural Stability and Dynamics. 2018;18(7): 1850099.

[19] Pan WF, Wang TR, Hsu CM. A curvature-ovalization measurement apparatus for circular tubes under cyclic bending. Experimental Mechanics. 1998;38(2):99-102.

(C) 2020 by the author(s). This work is licensed under a Creative Commons Attribution 4.0 International License (http://creativecommons.org/licenses/by/4.0/). Authors retain copyright of their work, with first publication rights granted to Tech Reviews Ltd. 